\title{
Small-angle x-ray scattering study of the structural evolution of the drying of sonogels with the liquid phase exchanged by acetone
}

\author{
D. R. Vollet, D. A. Donatti, A. Ibañez Ruiz, and H. Maceti \\ Department of Physics, IGCE-Universidade Estadual Paulista (UNESP), P.O. Box 178, CEP 13500-970 Rio Claro, São Paulo, Brazil
}

(Received 18 July 2003; revised manuscript received 27 October 2003; published 22 March 2004)

\begin{abstract}
The structural evolution on the drying of wet sonogels of silica with the liquid phase exchanged by acetone, obtained from tetraethoxisilane sonohydrolysis, was studied in situ by small-angle x-ray scattering (SAXS). The periods associated to the structural evolution as determined by SAXS are in agreement with those classical ones established on basis of the features of the evaporation rate of the liquid phase in the obtaining of xerogels. The wet gel can be described as formed by primary particles (microclusters), with characteristic length $a$ $\sim 0.67 \mathrm{~nm}$ and surface which is fractal, linking together to form mass fractal structures with mass fractal dimension $D=2.24$ in a length scale $\xi \sim 6.7 \mathrm{~nm}$. As the network collapses while the liquid/vapor meniscus is kept out of the gel volume, the mass fractal structure becomes more compacted by increasing $D$ and decreasing $\xi$, with smoothing of the fractal surface of the microclusters. The time evolution of the density of the wet gels was evaluated exclusively from the SAXS parameters $\xi, D$, and $a$. The final dried acetone-exchanged gel presents Porod's inhomogeneity length of about $2.8 \mathrm{~nm}$ and apparently exhibits an interesting singularity $D$ $\rightarrow 3$, as determined by the mass fractal modeling used to fit the SAXS intensity data for the obtaining of the parameters $\xi$ and $D$.
\end{abstract}

DOI: 10.1103/PhysRevB.69.094203

PACS number(s): $61.10 . \mathrm{Eq}, 61.43 . \mathrm{Hv}, 81.20 . \mathrm{Fw}, 82.70 . \mathrm{Gg}$

\section{INTRODUCTION}

A large variety of glass and glass ceramics has been obtained by sol-gel process from the hydrolysis of tetraethoxysilane (TEOS). ${ }^{1}$ Since water and TEOS are immiscible, a mutual solvent such as ethanol is usually employed as a homogenizing medium in the conventional sol-gel method. Because ethanol is a product of both the hydrolysis and the alcohol producing condensation reaction, sonochemistry is an alternative method to promote hydrolysis in the presence of the acid catalyst. ${ }^{2,3}$

The wet gel structure frequently exhibits a mass fractal feature ${ }^{4}$ consisting of a continuous solid network imbibed in a liquid phase, which can occupy until about 95 to $98 \%$ of the volume sample. ${ }^{5,6}$ Drying is the most critical step of the sol-gel processing for obtaining monoliths or if the textural properties of the wet gels are intended to be preserved. ${ }^{7} \mathrm{Su}-$ percritical drying (aerogels), freeze drying (criogels), and evaporation drying (xerogels) ${ }^{1}$ are the usual methods in dried gels production.

Particularly, the drying of xerogels exhibits three distinct stages: the constant rate period (CRP), the first falling rate period, and the second falling rate period. ${ }^{7}$ The drying stages can be observed experimentally by studying the rate of liquid loss versus the liquid content in the gel. The constant rate period, in which the greatest changes in volume, weight, and structure occur, is controlled by evaporation from the surface of the body and ends when shrinkage ceases. ${ }^{7,8}$ The rate of evaporation per unit area of the drying surface remains constant while the solid network collapses into the liquid, as a result of the compressive stress imposed by the tensile stress (capillary forces) of the liquid, and the liquid/vapor meniscus is kept at the drying surface. ${ }^{7}$ The first falling rate period starts when the network becomes strong enough to resist further shrinkage and the radius of meniscus reduces enough to enter the pore, which begins to empty. This stage is controlled by flow through small pores and liquid layers over the pore surface and only small changes in weight and volume are observed. ${ }^{8}$ In the second falling rate period, the liquid pathway to the surface becomes discontinuous and a very small loss of weight follows, until the equilibrium, by evaporation within the pores and diffusion of the vapor to the surface. ${ }^{8}$ It has been pointed that most alkoxide-derived gels may not exhibit a CRP since the liquid phase of the gel is a mixture of water and alcohol. ${ }^{7}$ Kawaguchi et al. ${ }^{9}$ found the evaporation rate to be initially constant for large pore silica gel, meanwhile Hench and Wilson ${ }^{8}$ did not find a constant rate of loss of weight during drying alkoxide-derived gels, even when normalized to the surface area.

In the present work, the liquid phase of wet sonogels obtained from the TEOS sonohydrolysis was exchange by acetone, in order to minimize the effects of the surface tension on the subsequent drying process in the obtaining of xerogels. The drying of the acetone-exchanged wet sonogel was studied in situ by small angle X-ray scattering (SAXS) at room temperature. Since the evaporation rate of acetone occurs relatively rapid, even at room temperature, the study allowed the drying stages and the corresponding structural evolution to be followed by SAXS. The method employed in the present work in studying the phenomenology of the drying of gels is not a direct method that allows the liquid/vapor meniscus movement (kept constant at the drying surface, reduction in meniscus radius, and its movement into the small pores, etc.) to be directly followed, but it is an indirect method that allows us to associate the structural evolutions, including contrast variations, with the well known drying stages.

\section{EXPERIMENT}

The samples were prepared from the sono-hydrolysis of mixtures of $25 \mathrm{ml}$ of tetraethoxysilane (TEOS), $8 \mathrm{ml}$ of dis- 
tilled and dionized water, and $5 \mathrm{ml}$ of $0.1 \mathrm{~N} \mathrm{HCl}$ as a catalyst. The resulting $\mathrm{pH}$ of the mixture was about 1.5-2.0. The hydrolysis was promoted during 10 min under a constant power $(60 \mathrm{~W})$ of ultrasonic radiation. Next, the sol was diluted in $14.3 \mathrm{ml}$ of water and sonication was continued for 2 min for complete homogenization. The $\mathrm{pH}$ of the resulting sol was adjusted to 4.5 by addition of $\mathrm{NH}_{4}(\mathrm{OH})$. The final water/TEOS molar ratio was equal to 14.4. The resulting sol was cast in sealed containers and kept under saturated conditions for 20 days at $40{ }^{\circ} \mathrm{C}$ for gelation and aging. The liquid phase of the sonogels was exchanged by pure acetone at room temperature. The volume of the acetone surrounding the gel, about ten times the apparent gel volume, was exchanged each $24 \mathrm{~h}$ during 10 days. For the SAXS measurements, a 2-mm-thick and 5-mm-diameter lamina of the acetone-saturated gel was placed in an open sample holder with two lateral $10-\mu \mathrm{m}$-thick Mylar sheets, just to avoid the drop of the sample, so that the evaporation process was free to take place practically from all external surface of the sample. The SAXS data were obtained as a function of the drying time at room temperature using synchrotron radiation with a wavelength $\lambda=0.1608 \mathrm{~nm}$. The beam was monochromatized by a silicon monochromator and collimated by a set of slits defining a pin-hole geometry. A one-dimensional position sensitive X-ray detector was used to record the SAXS intensity, $I(q)$, as function of the modulus of the scattering vector $q=(4 \pi / \lambda) \sin (\theta / 2)$, where $\theta$ is the scattering angle. The experimental set allowed to get SAXS data from $q_{0}$ $=0.19 \mathrm{~nm}^{-1}$ to $q_{m}=4.4 \mathrm{~nm}^{-1}$ with a resolution of $\Delta q$ $=3.36 \times 10^{-3} \mathrm{~nm}^{-1}$. The data were corrected by the parasitic scattering and the sample attenuation and normalized by the intensity of the incident beam. The sample attenuation, $A$, defined as the incident to the transmitted intensity ratio, was measured continuously along the drying time. The scattering intensity from a $45^{\circ}$-tilted Mylar film placed before the sample, as measured by a scintillation detector positioned perpendicularly to incident beam, was taken as a measure proportional to the incident beam intensity. The scattering intensity produced by the incidence of the transmitted beam on the beam stop of the SAXS detector, as measured by a second scintillation detector conveniently positioned at the proximities of the beam stop, was taken as a measure proportional to the transmitted beam intensity.

\section{INTERPRETATION OF THE SAXS DATA}

Fresh gels frequently exhibit mass fractal structures that can be recognized by typical power-law decrease on $q$ of the SAXS intensity in a $q$ range between $\xi \gg q^{-1} \gg a$, where $\xi$ is the characteristic length of the fractal structure and $a$ the characteristic length of the primary particles composing the structure. ${ }^{10}$ As found for the acetone-exchanged wet gel studied in this work, the SAXS intensity departs from the powerlaw regime at low- $q$ values due to the finite correlation range $\xi$ of the mass fractal structure, and at high- $q$ values due to the surface fractal characteristic of the primary particles (microclusters) composing the structure. We first analyze the SAXS intensity at the low- and medium- $q$ regions in which it can be fitted by ${ }^{11}$

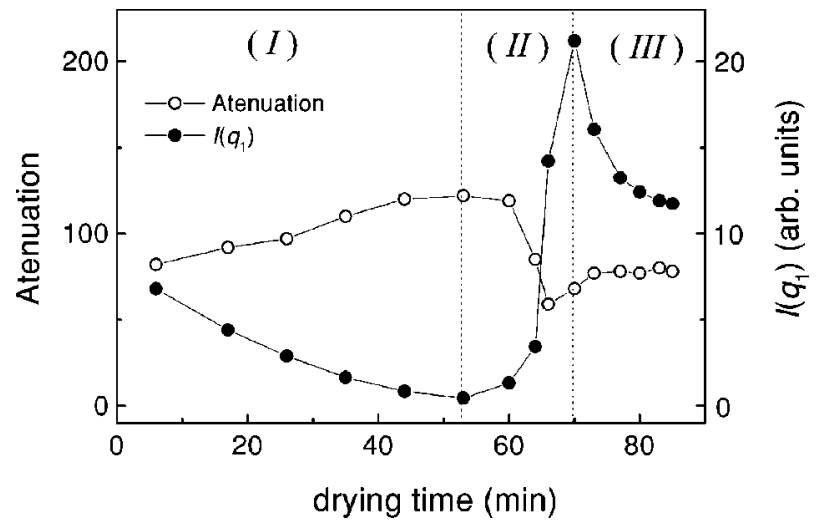

FIG. 1. Structural evolution periods as established by SAXS at room temperature on the drying of sonogels with the liquid phase exchanged by acetone: (I) network collapse period; (II) electronic density contrast variation period; (III) final microclusters structural evolution period.

$$
\begin{aligned}
I(q)= & B \sin [(D-1) \arctan (q \xi)] /\left(1+q^{2} \xi^{2}\right)^{(D-1) / 2} \\
& \times(D-1) q \xi,
\end{aligned}
$$

where $D$ is the mass fractal dimension of the structure, a physically acceptable value between $1<D<3$, and $B$ is a constant for a given $D$ and $\xi$.

At high- $q$ region, it was found the intensity crossing over to power-law decrease on $q$ as

$$
I(q) \sim q^{-\alpha},
$$

with the exponent $\alpha$ found between 3 and 4 . This behavior was interpreted as a property of the primary particle surfaces that would be fractal with dimensionality $D_{S}$ given by ${ }^{11}$

$$
D_{S}=6-\alpha .
$$

\section{RESULTS}

The experimental SAXS curves exhibited a continuous decrease in intensity at the first instants of the process. The diminution in intensity was found approximately $q$ independent, mainly within the low- $q$ range. We have then assumed that the intensity for a fixed and low $q_{1}$ value is a relative measure of the structural evolution of the system. Figure 1 shows the evolution with time of the SAXS intensity, $I\left(q_{1}\right)$, as measured at $q_{1}=0.22 \mathrm{~nm}^{-1}$, together with the sample attenuation along the drying process of the acetoneexchanged silica sonogel. Three distinct stages are apparent along the process: (I) diminution of $I\left(q_{1}\right)$ and increase of $A$, (II) increase of $I\left(q_{1}\right)$ and diminution of $A$, and (III) further diminution of $I\left(q_{1}\right)$ and constancy of $A$.

Stage I is attributed to the network collapse in which the shrinkage of the gel is responsible for the greatest structural modifications. This stage is associated to the constant rate period (CRP) frequently found in the drying of xerogels, in which the meniscus liquid/vapor is kept at the external surface of the gel unable to penetrate into the pores, while the gel collapses. Therefore, no contrast variation occurs during this stage, so that the two phases scattering condition is ob- 


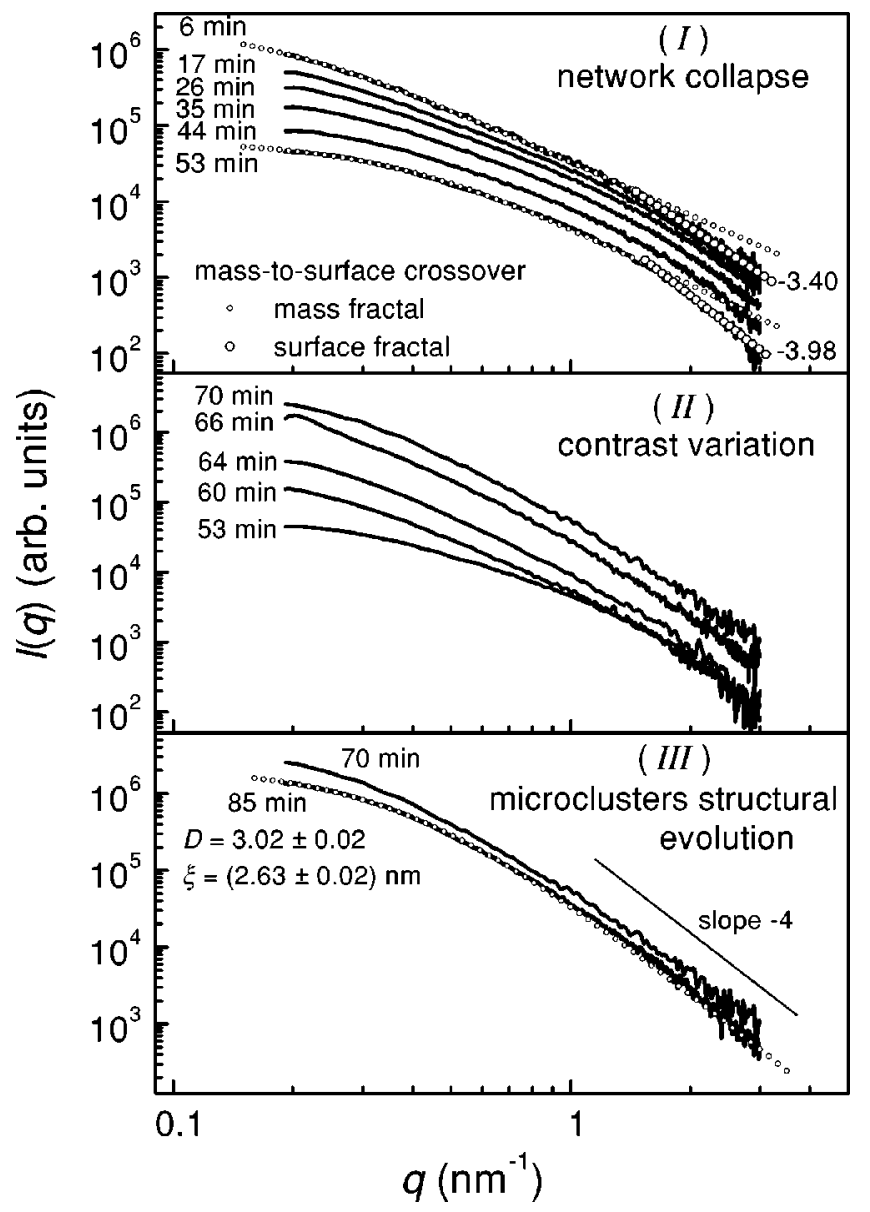

FIG. 2. SAXS intensity as a function of the modulus of the scattering vector $q$ with time along the periods of structural evolution as defined in Fig. 1. The small circle lines are examples of fitting of the mass fractal modeling [Eq. (1)] to the corresponding experimental data. Medium circle lines are examples of the fitting of the asymptotic power-law decaying [Eq. (2)] in the obtaining of the surface fractal dimension.

servable for further SAXS analysis.

Stage II is associated with the contrast variations due to the penetration of the meniscus liquid/vapor into the pore when the network collapse ceases, since the network becomes strong enough to resist further shrinkage. As the SAXS intensity is proportional to the square of the electronic density difference between the phases, we expect the intensity to increase with pore emptying. The emptying of the pores should start first by the big ones on view of the Kelvin equation relating the pore radius with the equilibrium vapor pressure under an evaporation process, in an analogy with the desorption branch in a gas adsorption experiment. The analysis of the SAXS intensity associated with systems with three electronic densities as found in this stage is very complex as considered when compared to the usual treatment applied to a two-phase system.

Stage III is associated, as we will later discuss, to further structural evolutions in the microclusters (or particles) of the sonogels.

Figure 2 shows the SAXS intensity as a function of the modulus of the scattering vector $q$ along of the time of the

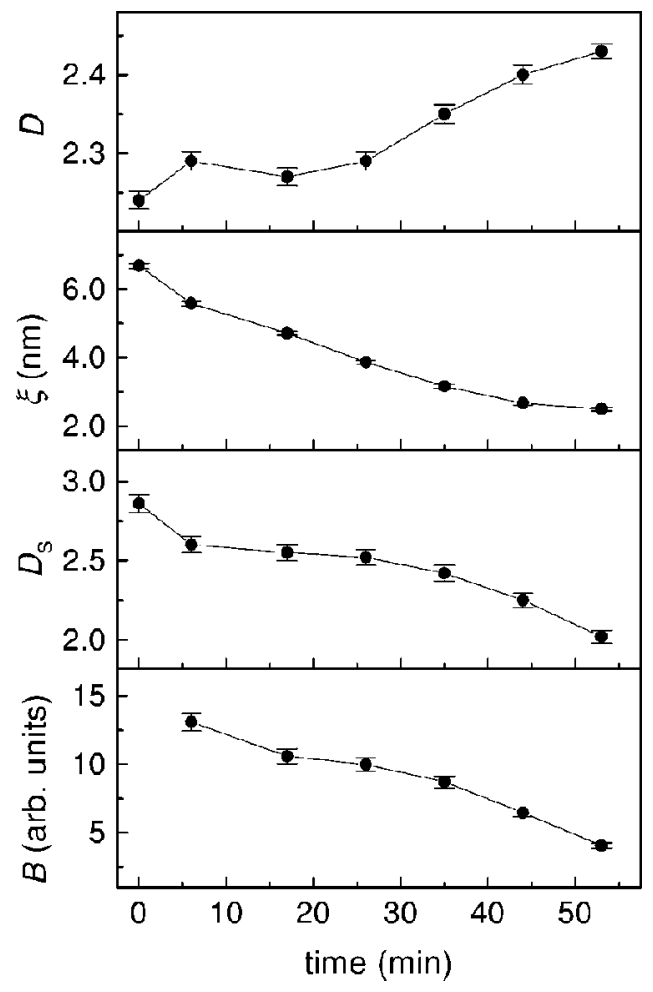

FIG. 3. Time evolution of the structural parameters obtained by SAXS for acetone-exchanged wet sonogels along the network collapse period.

three stages defined in Fig. 1. During the network collapse (stage I), the SAXS intensity curves as a function of modulus of the scattering vector $q$ are all practically parallel, the intensity diminishing with time. In addition, Eq. (1) fits well to the experimental data up to a $q$ value of about $q_{a}$ $\sim 1.5 \mathrm{~nm}^{-1}$, and for greater $q$ values, the intensity follows reasonably a power-law decrease given by Eq. (2), with the exponent $\alpha$ increasing from 3.4 to almost 4.0 during this stage. The mass-to-surface fractal crossover is attributed to the microclusters surface, which is fractal, so that it is becoming more smooth as the network is collapsing, since $\alpha$ is going to $4.0\left(D_{S} \rightarrow 2\right)$. The structural parameters of the mass fractal structure $(\xi$ and $D)$ and of the surface fractal of the microclusters $\left(D_{S}\right)$ are shown in Fig. 3 as a function of the network collapsing time. The diminution of $\xi$ and increase of $D$ is compatible with the compacting of the structure as the network collapses. Along the contrast variation stage II, it is apparent the emptying first of larger pores, since the intensity increases first at low $q$-region (see the 60 min-labeled sample compared to that labeled 53 min in Fig. 2). Further structural evolutions associated with stage III are attributed to the final compacting and smoothing of the microclusters (particles), since $D \rightarrow 3$ and $\alpha \rightarrow 4.0\left(D_{S} \rightarrow 2\right)$, as in a classical two-phase scattering system.

\section{DISCUSSION}

The structural transition from mass to surface fractal as suggested by the crossover found in the scattering intensity power-law regime at about $q_{a} \sim 1.5 \mathrm{~nm}^{-1}$, during stage I in 


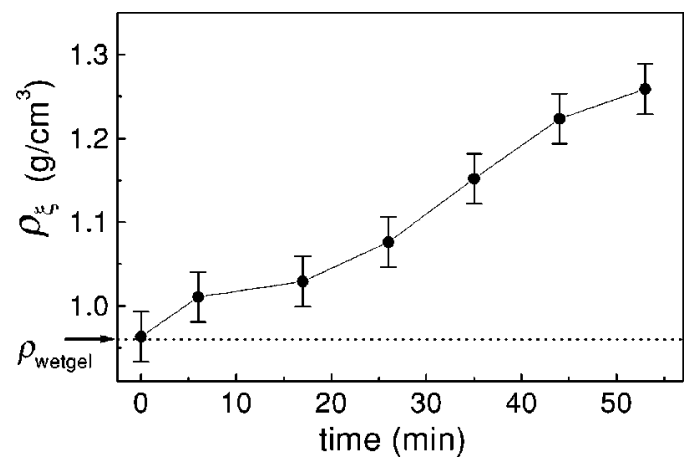

FIG. 4. Time evolution of the mass fractal structure density as evaluated by SAXS for acetone-exchanged wet sonogels along the network collapse period. The indicated value $\rho_{\text {wet gel }}$ corresponds to the measured density of an ethanol-exchanged wet sonogel.

which the network is collapsing in saturated conditions, can be interpreted as the following modeling: the wet gel is composed by primary particles (or microclusters) with characteristic length given by $a \sim 1 / q_{a} \sim 0.67 \mathrm{~nm}$, with extremely rough surface, at the beginning of drying, which link together to form mass fractal structures with characteristic length $\xi$ and mass fractal dimension $D$. This modeling is similar to that described by Vacher et al. ${ }^{11}$ in silica aerogels, inclusive with respect to the value of the crossover at $q_{a}$ $\sim 1.5 \mathrm{~nm}^{-1}$. Here, as the network collapses, the fractal structure becomes more compacted, decreasing $\xi$ and increasing $D$, and the surface of the microclusters becomes more smoothed. The network compacting is also responsible by the diminution of the SAXS intensity due to the increase of the interference between the scattered waves from different parts of the sample, as they are approximating together as the network collapses. The fractal structure evolution along network collapse can be followed by analyzing the time evolution of the density of the mass fractal structure, $\rho_{\xi}$, which can be evaluated exclusively from the SAXS parameters $\xi$, $D$ and $a$ through the equation ${ }^{11}$

$$
\Delta \rho_{\xi} / \Delta \rho_{\mathrm{a}}=(\xi / a)^{D-3},
$$

where $\Delta \rho_{\xi}=\rho_{\xi}-\rho_{\mathrm{L}}$ and $\Delta \rho_{\mathrm{a}}=\rho_{\mathrm{a}}-\rho_{\mathrm{L}}$, with $\rho_{\mathrm{L}}$ being the density of the liquid phase (acetone) and $\rho_{\mathrm{a}}$ the density of primary particles or microclusters. $\rho_{\mathrm{a}}$ was evaluated through Eq. (4) from the measured value $\rho_{\text {wetgel }}=0.96 \mathrm{~g} / \mathrm{cm}^{3}$, as the density obtained from weight and volume measurements of an ethanol-exchanged wet gel sample, since ethanol is less volatile and have the same density as acetone, and $D=2.24$ and $\xi=6.68 \mathrm{~nm}$, as determined by SAXS from Eq. (1) for such a sample. We have obtained $\rho_{\mathrm{a}}=1.78 \mathrm{~g} / \mathrm{cm}^{3}$, which is less than the value frequently quoted for the fused silica $\left(2.2 \mathrm{~g} / \mathrm{cm}^{3}\right)$, revealing some structure in the microclusters. Figure 4 shows the time evolution of $\rho_{\xi}$ as evaluated from Eq. (4) with $\rho_{\mathrm{a}}=1.78 \mathrm{~g} / \mathrm{cm}^{3}$ and $a=0.67 \mathrm{~nm}$. The values associated to zero time (Figs. 3 and 4) correspond all to those of the mentioned ethanol-exchanged wet gel sample. From the density data, the volume fraction of the liquid phase in the acetone-exchanged wet gel at the initial condition of dry- ing was evaluated as being about 0.83 and at the end of the stage of the network collapse as about 0.50 .

The diminution of the SAXS intensity associated to final of stage III, after the contrast variation stage, is attributed to further structural evolution in the microclusters, which would be contracting to density values greater than that $1.78 \mathrm{~g} / \mathrm{cm}^{3}$ obtained for the wet microclusters. If we assume that the final density of the microclusters is close to that of the fused silica $\left(2.2 \mathrm{~g} / \mathrm{cm}^{3}\right)$, then the volume of the microclusters would have been contracted by an additional quantity of about $19 \%$, so the pore volume fraction $(\phi)$ of the final dried gel would be about 0.59 .

The structural characteristics of the dried gel associated to the final of stage III were analyzed on basis of the same modeling as described by Eq. (1). Figure 2 (III) shows that Eq. (1) fits well the SAXS intensity data from the sample after $85 \mathrm{~min}$ of drying. Singularly, the fractal dimension $D$ was found to be approximately equal to 3 and the asymptotic approximation for the scattering intensity was found decaying proportional to $q^{-4}$, according to Porod's law.

The fact of the asymptotic scattering intensity to vanish or not as $D \rightarrow 3$ has been subject to some discussion. ${ }^{12}$ The small-angle scattering intensity from random fractals with $D \neq 3$ is known to decay proportional to $q^{-D}$, for $q \xi \gg 1$, where $\xi$ is the length of the overall dimension of the fractal. ${ }^{13}$ Surprisingly, Eq. (1) was found to be able to fit our experimental data with $D=3$ and describe the asymptotic scattering intensity according to Porod's law, i.e., decaying proportional to $q^{-4}$ instead $q^{-D}$. Indeed, a more careful inspection of Eq. (1) shows that, for $D=3, I(q)$ decays as $q^{-4}$ since $\sin [2 \arctan (q \xi)] \rightarrow \sin [2(\pi / 2-1 / q \xi)] \rightarrow 2 / q \xi$ as $q \xi$ $\gg 1$. Since Eq. (1) is properly applied to mass fractal structures, it is reasonable to realize that $D=3$ corresponds to homogeneous objects, in an overall length scale $\xi$, with perfectly smoothed surface, since $I(q) \sim q^{-4}$ for $q \xi \gg 1$. The surface per unit volume of such a dried sample, $S / V$, can be obtained from Porod's law ${ }^{14}$ through the equation

$$
S / V=4 \phi(1-\phi) / l_{P},
$$

where $\phi$ is the pore volume fraction $(\phi=0.59)$ and $l_{P}$ is the Porod's inhomogeneity length, which is obtained exclusively from SAXS data through the equation

$$
l_{P}=(4 / \pi) Q / K_{P}
$$

where $K_{P}$, the constant of Porod's law, is given by

$$
K_{P}=\lim _{q \rightarrow \infty} I(q) q^{4}
$$

and $Q$, the integrated intensity, as

$$
Q=\int_{0}^{\infty} q^{2} I(q) \mathrm{d} q .
$$

We have found $l_{P}=(2.79 \pm 0.16) \mathrm{nm}$ and $S / V=(0.35$ $\pm 0.02) \mathrm{nm}^{-1}$ for the dried sample, which corresponds to about $385 \mathrm{~m}^{2} / \mathrm{g}$, if we assume that silica particles with $\sim 2.2 \mathrm{~g} / \mathrm{cm}^{3}$ occupy the remaining 0.41 of the dried gel volume fraction. Monolithic xerogels, obtained through slow 
drying process from nonexchanged sonogels, frequently exhibit specific surface of about $390 \mathrm{~m}^{2} / \mathrm{g},{ }^{15}$ in good agreement with the value obtained for this acetone-exchanged sample submitted to rapid drying. The value of the Porod's inhomogeneity length $l_{P}=2.79 \mathrm{~nm}$ is also in agreement with the value of the overall length scale $\xi=2.63 \mathrm{~nm}$, as obtained by fitting Eq. (1) to the dried sample data [Fig. 2 (III)].

The feature $D=3$ together with the behavior $I(q) \sim q^{-4}$ for $q \xi \gg 1$ could also be interpreted as the scattering from a system with an extremely rough boundary space filling fractal surface, in a length scale less than $\xi \sim 2.7 \mathrm{~nm}$, as pointed by Rojanski et al. ${ }^{16}$ for mesoporous silica on basis of an integrated study combining adsorption, electronic energy transfer, and SAXS techniques. However, we think it would be a complex figure to be elucidated exclusively from our SAXS data.

\section{CONCLUSIONS}

The time structural evolutions on the drying of acetoneexchange silica sonogels, as determined by small angle x-ray scattering (SAXS), are in agreement with the classical periods established on basis of the features of the evaporation rate of the liquid phase in the obtaining of xerogels.

The wet gel can be described as formed by primary particles or microclusters, with density $\sim 1.78 \mathrm{~g} / \mathrm{cm}^{3}$, characteristic length $a \sim 0.67 \mathrm{~nm}$, and surface that is fractal, linking together to form mass fractal structures with mass fractal dimension $D=2.24$ in a length scale $\xi \sim 6.7 \mathrm{~nm}$.

As the network collapses during the constant rate period, in which the liquid/vapor meniscus is kept out of the gel volume, the mass fractal structure becomes more compacted by increasing $D$ and decreasing $\xi$, with smoothing of the fractal surface of the microclusters. The time evolution of the density of the wet gels was evaluated exclusively from the SAXS parameters $\xi, D$, and $a$.

The final dried acetone-exchanged gel exhibits Porod's inhomogeneity length of about $2.8 \mathrm{~nm}$ and specific surface whose value is in agreement with that of xerogels obtained by slow drying of nonexchanged sonogels.

\section{ACKNOWLEDGMENT}

Research was partially supported by LNLS-National Synchrotron Light Laboratory (Brazil) and FAPESP (Brazil).
${ }^{1}$ C. J. Brinker and G. W. Scherer, Sol-Gel Science: The Physics and Chemistry of Sol-Gel Processing (Academic, San Diego, 1990).

${ }^{2}$ M. Tarasevich, Am. Ceram. Soc. Bull. 63, 500 (1984).

${ }^{3}$ D.A. Donatti and D.R. Vollet, J. Non-Cryst. Solids 208, 99 (1996).

${ }^{4}$ D.R. Vollet, D.A. Donatti, and A. Ibañez Ruiz, J. Non-Cryst. Solids 288, 81 (2001).

${ }^{5}$ G.M. Pajonk, M. Reppelin-Lacroix, S. Abouarnadasse, J. Chaouki, and D. Klvana, J. Non-Cryst. Solids 121, 66 (1990).

${ }^{6}$ D.R. Vollet, D.A. Donatti, A. Ibañez Ruiz, and W.C. de Castro, Phys. Rev. B 67, 184207 (2003).

${ }^{7}$ G.W. Scherer, J. Non-Cryst. Solids 100, 72 (1988).

${ }^{8}$ L.L. Hench and M.J.R. Wilson, J. Non-Cryst. Solids 121, 234 (1990).
${ }^{9}$ T. Kawaguchi, J. Iura, N. Taneda, H. Hishikura, and Y. Kokubu, J. Non-Cryst. Solids 82, 50 (1986).

${ }^{10}$ D.W. Schaefer and K.D. Keefer, Phys. Rev. Lett. 53, 1383 (1984).

${ }^{11}$ R. Vacher, T. Woignier, J. Pelous, and E. Courtens, Phys. Rev. B 37, 6500 (1988).

${ }^{12}$ P. Pfeifer and P.W. Schmidt, Phys. Rev. Lett. 60, 1345 (1988).

${ }^{13}$ P.W. Schmidt and Xie Dacai, Phys. Rev. A 33, 560 (1986).

${ }^{14}$ G. Porod, in Small Angle X-ray Scattering, edited by O. Glatter and O. Kratky (Academic, London, 1982).

${ }^{15}$ D.R. Vollet, D.A. Donatti, and A. Ibañez Ruiz, J. Non-Cryst. Solids 306, 11 (2002).

${ }^{16}$ D. Rojanski, D. Huppert, H.D. Bale, Xie Dacai, P.W. Schmidt, D. Farin, A. Seri-Levy, and D. Avnir, Phys. Rev. Lett. 56, 2505 (1986). 